\title{
Differences in regional air trapping in current smokers with normal spirometry
}

\author{
Reza Karimi ${ }^{1,3}$, Göran Tornling (1) ${ }^{1}$, Helena Forsslund ${ }^{1}$, Mikael Mikko ${ }^{1}$, \\ Åsa M. Wheelock ${ }^{1}$, Sven Nyrén ${ }^{2,3}$ and C. Magnus Sköld ${ }^{1,3}$ \\ Affiliations: ${ }^{1}$ Dept of Medicine and Centre for Molecular Medicine (CMM) Respiratory Medicine Unit, \\ Karolinska Institutet, Karolinska University Hospital Solna, Stockholm, Sweden. ${ }^{2}$ Dept of Molecular Medicine \\ and Surgery, Division of Radiology, Karolinska Institutet, Karolinska University Hospital Solna, Stockholm, \\ Sweden. ${ }^{3}$ These authors contributed equally.
}

Correspondence: Reza Karimi, Dept of Medicine Solna, Karolinska Institutet, Lung-Allergy Clinic N2:02, Karolinska University Hospital Solna, SE 17176 Stockholm, Sweden. E-mail: reza.karimiaki.se

@ERSpublications

Smokers with regional air trapping on expiratory CT scan are less obstructive/have less emphysema than those without http://ow.ly/96lV305tgtZ

Cite this article as: Karimi R, Tornling G, Forsslund H, et al. Differences in regional air trapping in current smokers with normal spirometry. Eur Respir J 2017; 49: 1600345 [https://doi.org/10.1183/13993003.003452016].

ABSTRACT We investigated regional air trapping on computed tomography in current smokers with normal spirometry. It was hypothesised that presence of regional air trapping may indicate a specific manifestation of smoking-related changes.

40 current smokers, 40 patients with chronic obstructive pulmonary disease (COPD), and 40 healthy never- smokers underwent computed tomography scans. Regional air trapping was assessed on endexpiratory scans and emphysema, micronodules and bronchial wall thickening on inspiratory scans. The ratio of expiratory and inspiratory mean lung attenuation (E/I) was calculated as a measure of static (fixed) air trapping.

Regional air trapping was present in $63 \%$ of current smokers, in $45 \%$ of never smokers and in $8 \%$ of COPD patients $(\mathrm{p}<0.001)$. Current smokers with and without regional air trapping had E/I ratio of 0.81 and 0.91 , respectively $(\mathrm{p}<0.001)$. Forced expiratory volume in $1 \mathrm{~s}(\mathrm{FEV} 1)$ was significantly higher and emphysema less frequent in current smokers with regional air trapping.

Current smokers with regional air trapping had higher FEV1 and less emphysema on computed tomography. In contrast, current smokers without regional air trapping resembled COPD. Our results highlight heterogeneity among smokers with normal spirometry and may contribute to early detection of smoking related structural changes in the lungs.

Received: July 112015 | Accepted after revision: Oct 132016

Support statement: Supported by the Swedish Heart-Lung Foundation, the King Oscar II Jubilee Foundation, the Mats Kleeberg Foundation, King Gustaf V and Queen Victoria's Freemasons Foundation, Sandoz A/S, EU FP6 Marie Curie IRF, Swedish Foundation for Strategic Research (SSF), VINNOVA, Karolinska Institutet and through the Regional Agreement on Medical Training and Clinical Research (ALF) between Stockholm County Council and Karolinska Institutet. Funding information for this article has been deposited with the Open Funder Registry. The funders had no role in study design, data collection and analysis, decision to publish, or preparation of the manuscript.

Conflict of interest: None declared.

Copyright OERS 2017 


\section{Introduction}

Repetitive exposure to cigarette smoke causes epithelial damage and an inflammatory response, which gradually leads to structural changes in the airways and lung parenchyma. These structural changes, "remodelling", are the hallmarks of chronic obstructive pulmonary disease (COPD). It is now generally accepted that the small airways with diameter less than $2 \mathrm{~mm}$ are the primary area for early obstruction $[1,2]$. Small airways inflammation is considered to precede tissue destruction and development of emphysema [3]. Due to increased branching of the airways and subsequent expansion of the total cross-sectional lumen area, the small airways account for only $10-15 \%$ of the total airway resistance [4]. This area is sometimes referred as a "silent zone" because the disease cannot yet be measured by spirometry in an early stage and the patients do not experience significant clinical symptoms until substantial damage to the lungs has occurred [5].

The employment of computed tomography offers opportunity for early evaluation of subclinical, smoking-related pathological changes in the airways and lung parenchyma [6-8]. These early changes, distinguishable on inspiratory computed tomography scans as "tree in bud" and micronodules, gradually progress and lead to detachment of alveolar walls from the respiratory bronchiole to develop centrilobular emphysema [9]. Regional air trapping due to retention of air in the distal part of the respiratory tract appears, on expiratory computed tomography scans, as low attenuation patchy areas, which is regarded as a surrogate for small airways disease [10-12].

Quantification of pulmonary emphysema on computed tomography as volume of voxels below the threshold of -950 Hounsfield units is well established $[13,14]$. The ratio of mean lung attenuation on paired expiratory and inspiratory scans has been considered to quantify air trapping and has been proposed as an indicator for small airways disease [15-17]. In COPD and asthma, the ratio has been shown to be increased $[18,19]$. However, since the attenuation-based measurements are influenced by both hyperinflation and airways obstruction, visual assessment of regional air trapping on expiratory computed tomography scans may indicate a different finding [10].

This study was undertaken to compare visual assessments of early lungs lesions on computed tomography, with quantitative measurements and to signs of local and systemic inflammation. We hypothesised that a combination of quantitative lung attenuation measurements and visual assessment of morphological abnormality, i.e. regional air trapping on expiratory computed tomography scans and presence of micronodules, emphysema and bronchial wall thickening on inspiratory computed tomography, would reveal early pulmonary changes in smokers with normal spirometry. Furthermore, we performed bronchoscopy with bronchoalveolar lavage in order to characterise the inflammatory response in the distal airways, to reveal a potential link between imaging and smoking induced pathobiology.

\section{Materials and methods \\ Subjects}

The study was conducted as a part of the Karolinska COSMIC (COPD Smoking Proteomic) study, ClinicalTrials.gov identifier NCT02627872, [8, 20, 21]. Briefly, 45-65-year-old healthy never-smokers $(\mathrm{n}=40)$, Current smokers (hereafter referred as smokers) with normal spirometry $(\mathrm{n}=40)$ and COPD patients, Global Initiative for Chronic Obstructive Lung (GOLD) I and II ( $n=40)$ were included. Detailed demographic characteristics of the investigated groups have been published previously $[8,20]$ and a summary is shown in table 1. Of the COPD patients, 28 were current smokers and 12 were ex-smokers (smoking cessation $>2$ years). A medical examination and a standard chest radiograph were performed. Subjects with any significant medical condition, including asthma and allergy were not included. Inhaled or oral corticosteroids and exacerbation or airways infection during at least 3 months prior to inclusion were not allowed.

\section{Lung function}

Forced expiratory volume in $1 \mathrm{~s}$ (FEV1) and forced vital capacity (FVC) were measured 20 min after bronchodilatation with two inhalations of $0.5 \mathrm{mg}$ terbutalin (Bricanyl Turbuhaler; AstraZeneca, Södertälje, Sweden) using a spirometer (Vmax 229-6200 Legacy, Viasys, Yorba Linda, CA, USA). Total lung capacity and residual volume were measured by body plethysmography. Transfer factor of the lungs for carbon monoxide (TLCO) was measured by the single-breath method. Lung function data, including standardised residuals, i.e. Z-scores are presented in tables 1 and 2. Lower limits of normal (LLN) for FEV1/FVC, FVC and FEV1 were calculated, using GLI-2012 references [22] and the equations of the European Community of Coal and Steel (ECCS) [23] were used calculating static lung volumes and TLCO. All never smokers and smokers with normal spirometry had FEV1/FVC > LLN. Two females and five males with COPD, had $\mathrm{FEV} 1 / \mathrm{FVC}>\mathrm{LLN}$. However, the ratio of $\mathrm{FEV} 1 / \mathrm{FVC}$ was $<0.70$ which was the inclusion criteria for the study. The other COPD patients had FEV1/FVC $<$ LLN. 
TABLE 1 Demographic, lung function, quantitative and morphological computed tomography measurements for never smokers, smokers and COPD patients



Data are presented as mean $\pm \mathrm{SD}$, mean (range) or $\mathrm{n}(\%)$, unless otherwise stated. COPD: chronic obstructive pulmonary disease; FEV1: forced expiratory volume in $1 \mathrm{~s}$; FVC: forced vital capacity; TLC: total lung capacity; RV: residual volume; TLco: transfer factor of the lung for carbon monoxide; HU: Hounsfield units; LA-950: percentage of lung volume with attenuation <-950 HU. Current smoking is defined as the number of cigarettes per day for the last 6 months and $\mathrm{E} / \mathrm{I}$ ratio is the ratio between mean lung attenuation at expiration and inspiration. ": only current smokers COPD; ${ }^{\#}: p \leqslant 0.05,{ }^{\# \#}: p \leqslant 0.01$ and ${ }^{\# \# \#}: p \leqslant 0.001$ for comparison between never-smokers and smokers; ${ }^{+}: p \leqslant 0.05,{ }^{++}: p \leqslant 0.01$ and ${ }^{+++}: p \leqslant 0.001$ for comparison between smokers and COPD; and ${ }^{\S}: p \leqslant 0.05, \S \S: p \leqslant 0.01$ and ${ }^{\S \S}: p \leqslant 0.001$ for comparison between COPD and never smokers.

\section{Computed tomography imaging}

Multi-detector helical computed tomography examinations, without any contrast medium, were performed using a Siemens Somatom Sensation 16 slice CT (Siemens, Erlangen, Germany). Scans were performed in a supine position and covered the entire thorax. The scanning was performed with $16 \times 0.75 \mathrm{~mm}$ collimation, $120 \mathrm{kV}$, rotation time $0.5 \mathrm{~s}$ and pitch 1.5 . Dose modulation was used resulting in a mean of approximately $180 \mathrm{~mA}$. Subjects were then guided to hold their breath at end-expiration and sequential scans were obtained with $10 \mathrm{~mm}$ intervals. Axial images with $1 \mathrm{~mm}$ thickness and $0.5 \mathrm{~mm}$ overlap were reconstructed with a medium-high spatial resolution algorithm (B60f) in a $512 \times 512$ matrix. The end-expiratory scans were reconstructed as single slices in a similar way.

\section{Quantitative image analysis}

As previously described [8], computed tomography images were analysed using a semiautomatic programme (Volume, Siemens, Erlangen Germany). Attenuation values between -300 and $-1024 \mathrm{HU}$ were considered to isolate the lung parenchyma from surrounding and mediastinal structures. Mean lung attenuation and lung volume were measured automatically on both expiratory and inspiratory scans. The ratio of expiratory and inspiratory mean lung attenuation was calculated and designated "E/I" [17, 24]. The volumes of pixels with lower attenuation than $-950 \mathrm{HU}$ were also calculated on inspiratory scans [13].

\section{Morphological image analysis}

All scans were assessed independently and blinded in random order by two experienced reviewers (S. Nyren and R. Karimi). In a few occasions, the final interpretations were achieved by consensus. Regional air trapping typically appears on end-expiratory scans as patchy areas with lower attenuation than the 
TABLE 2 Comparison between two subgroups of smokers with and without regional air trapping on end-expiratory computed tomography

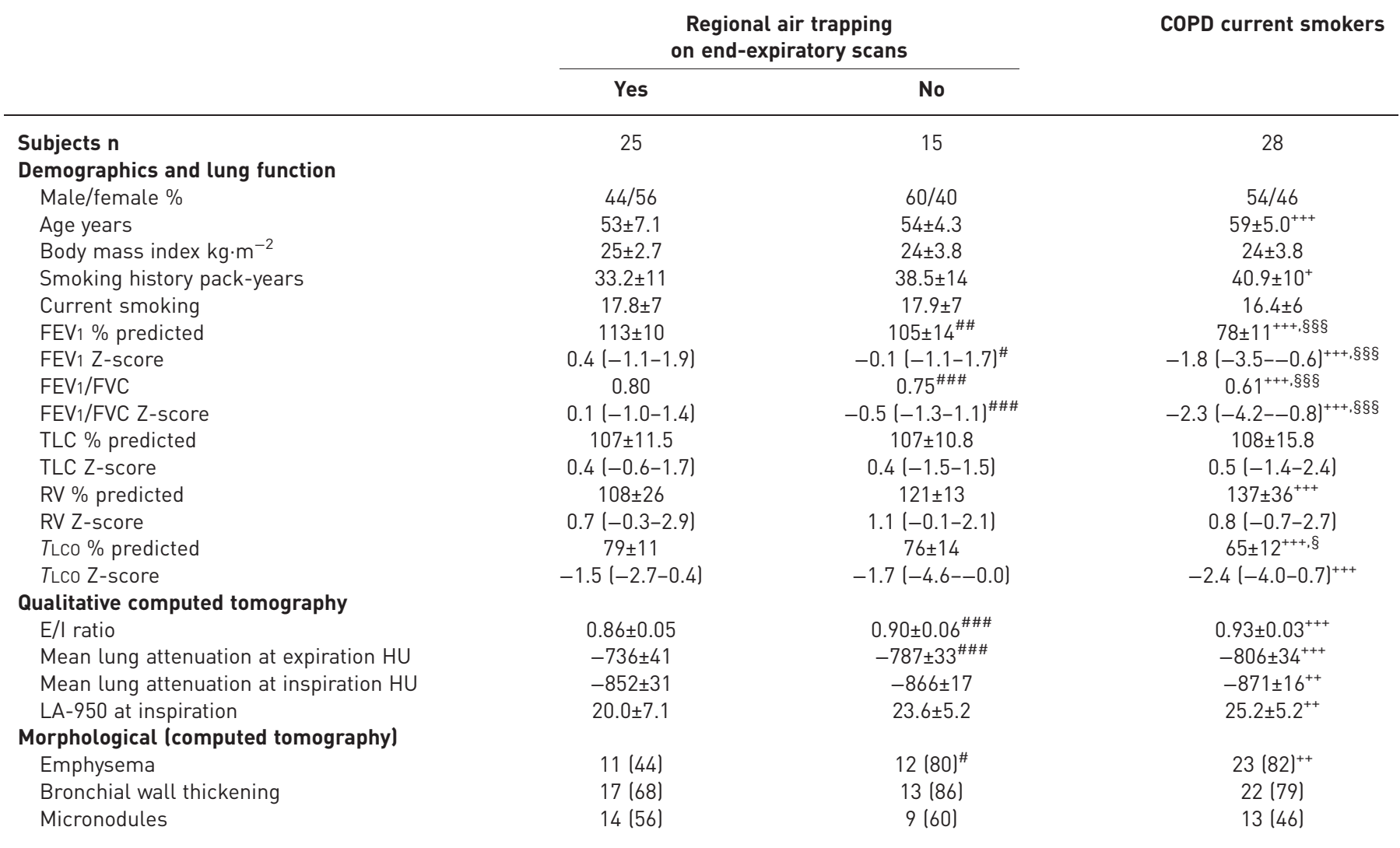

Data are presented as mean \pm SD, mean (range) or $\mathrm{n}(\%)$, unless otherwise stated. COPD: chronic obstructive pulmonary disease; FEV1: forced expiratory volume in $1 \mathrm{~s}$; FVC: forced vital capacity; TLC: total lung capacity; RV: residual volume; TLCo: transfer factor of the lung for carbon monoxide; $\mathrm{HU}$ : Hounsfield units. Current smoking is defined as the number of cigarettes per day for the last 6 months and $\mathrm{E} / \mathrm{I}$ ratio is the ratio

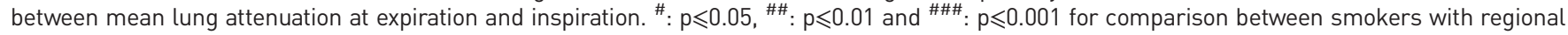
air trapping and smokers without regional air trapping; ${ }^{+}: p \leqslant 0.05,{ }^{++}: p \leqslant 0.01$ and ${ }^{+++}: p \leqslant 0.001$ for comparison between COPD and smokers with regional air trapping; and ${ }^{\S}: p \leqslant 0.05$ and ${ }^{\S \S}$ : $p \leqslant 0.001$ for comparison between COPD and smokers without regional air trapping.

surrounding tissues. In the current study, regional air trapping was considered present when at least three secondary lobules were involved (figure 1). Emphysema was evaluated on inspiratory scans dichotomously and considered to be present when $>5 \%$ of the whole lung parenchyma was involved. Bronchial wall thickening was considered to be present when both central bronchi and peripheral wall thickening were present simultaneously. Micronodules were identified as ill-defined small $(2-3 \mathrm{~mm})$ parenchymal ground glass attenuation areas distinguishable on inspiratory CT scans (figure 2).

FIGURE 1 Expiratory thin-section computed tomography in a 50-year-old male smoker with normal lung function, demonstrating regional air trapping as patchy area of low attenuation located mainly at dependent area of the right and left lower lobes (arrows).

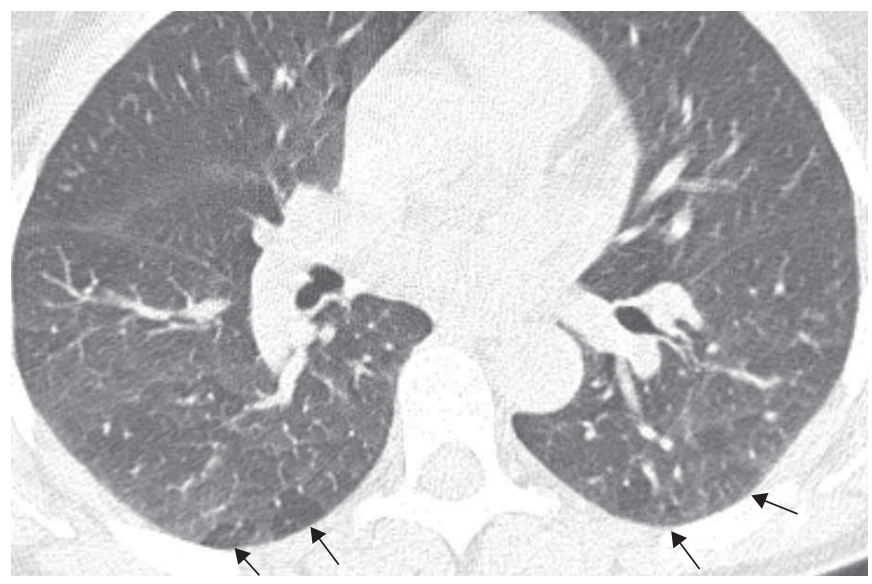


FIGURE 2 Axial inspiratory thin-section computed tomography of a 48-year-old female smoker with normal spirometry. At the level of the upper lobes micronodules can be visualised as indistinct and illdefined ground glass attenuation. These are more abundant at the anterior and central part of left upper lobe. Bronchial wall thickening is also present.

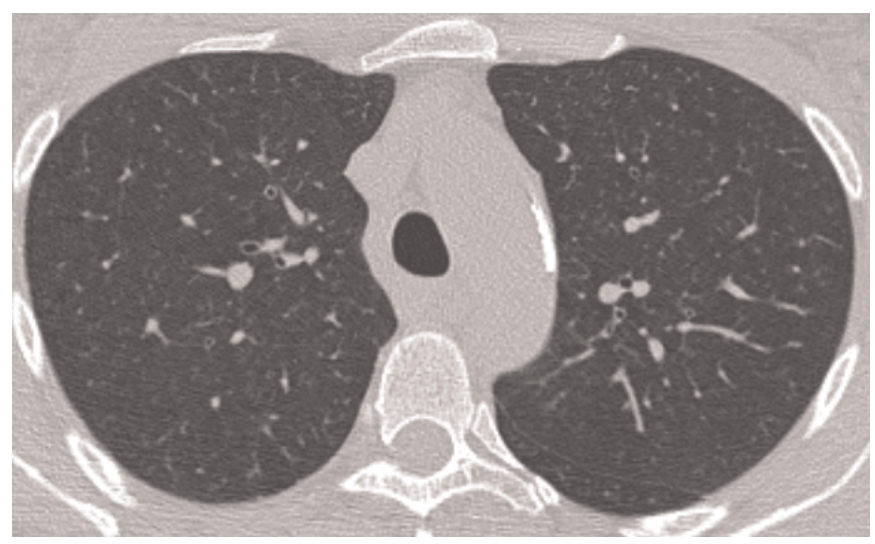

\section{Bronchoscopy and bronchoalveolar lavage}

Bronchoscopy with bronchoalveolar lavage (BAL) was performed in order to obtain cells from the distal airways. Bronchoscopy procedure and preparation of BAL cells were performed according to a standard procedure in our department, described in detail previously [25]. Immunofluorescent surface staining and flow cytometry were used to characterise the major lymphocyte subsets described in detail in previous study [21].

\section{Statistical analysis}

Unpaired two-tailed t-tests were employed comparing normally distributed data between two groups. One way ANOVA with Bonferroni adjustment for multiple comparisons was utilised for univariate statistical testing between groups. The values were expressed as mean \pm SD. Weighted kappa scores and percentage of agreement for each computed tomography morphological finding were calculated between the two reviewers. Univariate linear regression was employed to evaluate relationship between E/I with lung function, age, body mass index (BMI) and smoking. Binary variables were analysed with logistic regression model. Statistical significances were defined as p-values $<0.05$. All statistical analysis were performed using Stata12 software (Stata Corp, College Station, TX, USA).

\section{Results}

\section{Quantitative findings on computed tomography}

Quantitative computed tomography indices are shown in table 1. Mean lung attenuation at expiration was significantly higher for smokers compared with never smokers. The mean \pm SD ratio of expiratory and inspiratory mean lung attenuation "static air trapping" (E/I), was significantly lower in smokers $(0.88$ $\pm 0.05)$ and never smokers $(0.89 \pm 0.04)$, compared with COPD patients $(0.93 \pm 0.05)(\mathrm{p}<0.001$ for both compared with COPD), (figure 3). Percentage of lung volume at inspiration with voxels lower than

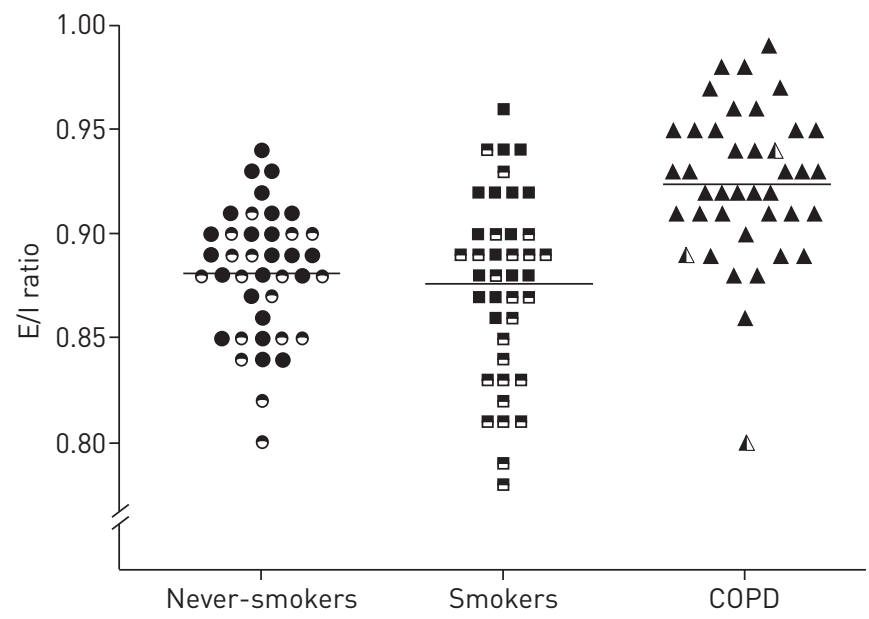

FIGURE 3 Ratio between mean lung attenuation at expiration and inspiration ( $E / /$ ratio) in never smokers, smokers and chronic obstructive pulmonary disease (COPD) patients. Half-filled symbols represent individuals with regional air trapping and filled symbols indicate individuals without regional air trapping. Note that smokers seems to be divided in two different subgroups based on the presence of regional air trapping while the never smokers are mixed homogenously. 
-950 HU was significantly lower in smokers compared with both the never smokers and the COPD patients $(\mathrm{p}<0.001$ for both). When the COPD patients were divided into current and ex-smokers, the latter group had higher percentage of lung volume at inspiration with voxels lower than $-950 \mathrm{HU}(33.2 \pm 6.2$ versus $25.2 \pm 5.2)(\mathrm{p}<0.001)$. There were no sex differences (data not shown).

\section{Morphological findings on computed tomography}

Morphological findings are illustrated in table 1. Regional air trapping was present in $63 \%$ of the smokers, in $45 \%$ of the never smokers and in $8 \%$ of the COPD patients $(\mathrm{p}<0.001$ for both compared to COPD). The difference between smokers and never smokers was not statistically significant. Emphysema was not present in the never smokers but in $55 \%$ of the smokers and in $80 \%$ of the COPD patients; $(\mathrm{p}<0.001$ for both compared to never smokers). Bronchial wall thickening was found in $75 \%$ of the smokers, in $12 \%$ of the never smokers and in $80 \%$ of COPD patients, $(\mathrm{p}<0.001$ for both compared to never smokers). Micronodules were detected in $58 \%$ of the smokers which was significantly higher than COPD patients (38\%; $\mathrm{p}<0.05)$. Micronodules were absent in never smokers. Solid nodules larger than $3 \mathrm{~mm}$ were found in all three groups without any significant difference (table 1).

\section{Inter-observer agreement}

Agreement between the two reviewers and the kappa score for regional air trapping was $83 \%$ and 0.66 in smokers and $80 \%$ and 0.60 in never smokers, respectively. The numbers of COPD with regional air trapping were too low to admit statistical calculations. Agreement and kappa score for presence of emphysema in smokers was $93 \%$ and 0.85 and in COPD $97 \%$ and 0.89 ; for micronodules, in smokers it was $75 \%$ and 0.5 and in COPD $86 \%$ and 0.68 ; and for bronchial wall thickening, in smokers was $55 \%$ and 0.17 and in COPD $71 \%$ and 0.37 .

\section{Comparison between smokers with or without regional air trapping}

Smokers were divided in two groups according to presence $(n=25)$ or absence $(n=15)$ of regional air trapping on end expiratory computed tomography scans. This division was based on the hypothesis that smokers with and without regional air trapping may differ in their inflammatory response and other smoking-related changes.

The two subgroups were similar regarding age, BMI, smoking history and current smoking status (table 2). Smokers without regional air trapping had significantly lower FEV1 and FEV1/FVC ratio $(p<0.01$ and $\mathrm{p}<0.001$, respectively). They also had lower mean lung attenuation at expiration $(\mathrm{p}<0.001)$, a higher E/I ratio $(0.90 \pm 0.060$ versus $0.86 \pm 0.05 ; \mathrm{p}<0.001)$, and more emphysema $(80 \%$ versus $44 \% ; \mathrm{p}<0.05)$, compared with smokers with regional air trapping (figure 3 and table 2). Micronodules were found, in logistic regression model, more frequently in smokers with emphysema (odds ratio 6.8, 95\% CI 1.7-27; $\mathrm{p}<0.01)$.

\section{Regional air trapping in smokers: comparison with COPD}

E/I ratio and the percentage of lung volume with attenuation <-950 HU (LA-950) at inspiration differed significantly between COPD and smokers with regional air trapping but not between COPD and smokers without regional air trapping (table 2). For morphological indices, emphysema was significantly more common in smokers without regional air trapping (82\%) and in COPD (80\%) compared with smokers with regional air trapping (44\%).

\section{Associations between BAL findings and computed tomography indices}

The percentage of BAL fluid recovered was significantly $(\mathrm{p}<0.01)$ higher in smokers with regional air trapping $(62.0 \pm 9.0 \%)$ compared both with smokers without regional air trapping $(53 \pm 10 \%)$ and with COPD patients $(45 \pm 12 \%)$, (table 3 ). The total cell yield in BAL was significantly higher in smokers with regional air trapping compared both to smokers without regional air trapping $(\mathrm{p}<0.01)$ and COPD $(\mathrm{p}<0.001)$. There were no differences regarding cell concentration and differential cell counts in BAL between the two smokers groups. The percentage of $\mathrm{CD}^{+} \mathrm{T}$-lymphocytes was significantly lower $(46 \pm 13 \%$ and $62 \pm 15 \% ; \mathrm{p}<0.01)$ and conversely, the percentage of $\mathrm{CD} 8^{+} \mathrm{T}$-lymphocytes was significantly higher $(45$ $\pm 13 \%$ and $29 \pm 15 \%$; $<<0.01$ ) in smokers with bronchial wall thickening compared with those without (data not shown).

\section{Correlations between static air trapping (E/I) and BAL findings}

In a multivariate model, after correction for age, BMI and smoking, a negative correlation between $\mathrm{E} / \mathrm{I}$ and neutrophil concentration in BAL in smokers was found (figure 4) $(\mathrm{r}=-0.47$ and $\mathrm{p}<0.001)$. While mean lung attenuation at expiration was positively correlated to neutrophil concentration, $(r=0.65$ and $p<0.001)$, 
TABLE 3 Measurements of local and systemic inflammation on two subgroups of smokers, with and without regional air trapping, data on COPD current smokers are also presented

\begin{tabular}{|c|c|c|c|}
\hline & \multicolumn{2}{|c|}{$\begin{array}{l}\text { Smokers with regional air } \\
\text { trapping on expiratory scans }\end{array}$} & \multirow[t]{2}{*}{$\begin{array}{c}\text { COPD } \\
\text { current smokers }\end{array}$} \\
\hline & Yes & No & \\
\hline Subjects $\mathrm{n}$ & 25 & 15 & 28 \\
\hline Male/female \% & $44 / 56$ & $60 / 40$ & $54 / 46$ \\
\hline BAL recovery $\%$ & $62.0 \pm 9.0$ & $53 \pm 10^{\#}$ & $45 \pm 12^{+++}$ \\
\hline Total cell yield $\times 10^{6}$ & $89.2 \pm 48$ & $66.6 \pm 27^{\#}$ & $53.5 \pm 34^{++}$ \\
\hline BAL cell concentration $10^{6} \cdot \mathrm{L}^{-1}$ & $578 \pm 303$ & $519 \pm 164$ & $493 \pm 255$ \\
\hline BAL macrophages $10^{6} \cdot \mathrm{L}^{-1}$ & $557 \pm 291$ & $500 \pm 157$ & $471 \pm 250$ \\
\hline BAL lymphocytes $10^{6} \cdot \mathrm{L}^{-1}$ & $14.0 \pm 11$ & $14.3 \pm 13$ & $13.1 \pm 9.0$ \\
\hline BAL neutrophils $10^{6} \cdot \mathrm{L}^{-1}$ & $5.3 \pm 6.2$ & $3.5 \pm 3.1$ & $4.7 \pm 4.1$ \\
\hline BAL eosinophils $10^{6} \cdot \mathrm{L}^{-1}$ & $1.5 \pm 3.5$ & $1.4 \pm 2.4$ & $3.5 \pm 9.7$ \\
\hline Percentage of $\mathrm{CD}^{+} \mathrm{T}$-lymphocytes & $54 \pm 17$ & $44 \pm 18$ & $50.0 \pm 22$ \\
\hline Percentage of $\mathrm{CD}^{+} \mathrm{T}$-lymphocytes & $37 \pm 16$ & $48 \pm 18$ & $43 \pm 23$ \\
\hline Ratio of $\mathrm{CD4}^{+}$and $\mathrm{CD}^{+}$ & $2.2 \pm 2.5$ & $1.5 \pm 2.1$ & $2.1 \pm 2.3$ \\
\hline White blood cell counts $10^{9} \cdot \mathrm{L}^{-1}$ & $7.4 \pm 1.6$ & $7.5 \pm 1.6$ & $8.0 \pm 1.9$ \\
\hline Serum high sensitive-CRP $\mathrm{g} \cdot \mathrm{L}^{-1}$ & $2.1 \pm 2.1$ & $1.6 \pm 1.0$ & $3.1 \pm 3.0$ \\
\hline Serum orosomucoid $\mathbf{g} \cdot \mathbf{L}^{-1}$ & $0.83 \pm 0.20$ & $0.71 \pm 0.12$ & $3.8 \pm 4.0$ \\
\hline Serum haptoglobin $\mathbf{g} \cdot \mathbf{L}^{-1}$ & $1.3 \pm 0.53$ & $1.0 \pm 0.40$ & $1.5 \pm 0.45^{\S \S}$ \\
\hline Serum $\operatorname{Ig} \mathbf{G} \mathbf{g} \cdot \mathbf{L}^{-1}$ & $8.7 \pm 1.7$ & $9.9 \pm 1.9^{\#}$ & $9.4 \pm 1.7$ \\
\hline Serum $\operatorname{Ig} \mathbf{A} \mathbf{g} \cdot \mathbf{L}^{-1}$ & $2.1 \pm 0.89$ & $2.3 \pm 0.80$ & $2.4 \pm 0.83$ \\
\hline
\end{tabular}

Data are presented as mean $\pm S D$, unless otherwise stated. COPD: chronic obstructive pulmonary disease; BAL: bronchoalveolar lavage; CRP: C-reactive protein. ${ }^{\#}: p \leqslant 0.05$ for comparison between smokers with regional air trapping and smokers without; ${ }^{++}: p \leqslant 0.01$ and ${ }^{+++}: p \leqslant 0.001$ for comparison between COPD and smokers with regional air trapping; and ${ }^{\S \S}: \mathrm{p} \leqslant 0.01$ for comparison between COPD and smokers without regional air trapping.

there were no correlations to any other cell types in BAL neither to inflammatory parameters in serum (data not shown).

Correlation between residual lung-volume on plethysmography with expiratory volume on computed tomography

Agreement between lung volume measured with computed tomography, at expiration, and residual volume measured with plethysmography is illustrated graphically in figure 5 for all subjects; correlation coefficient was $0.72(\mathrm{p}<0.001)$.

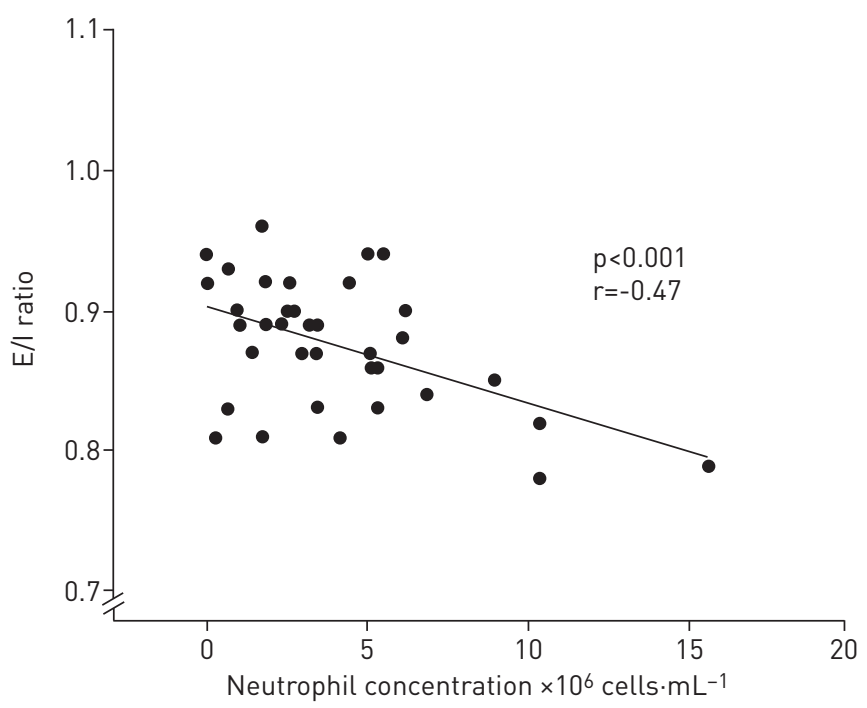

FIGURE 4 Correlation between neutrophil concentration (cells $\times 10^{6} \cdot \mathrm{L}^{-1}$ ) in broncho-alveolar lavage, and ratio between mean lung attenuation at expiration and inspiration (E/I-ratio; "static air trapping"), in smokers with normal spirometry. 
FIGURE 5 Correlation between lung volume at end expiration measured with computed tomography (CT) and residual volume measured with plethysmography.

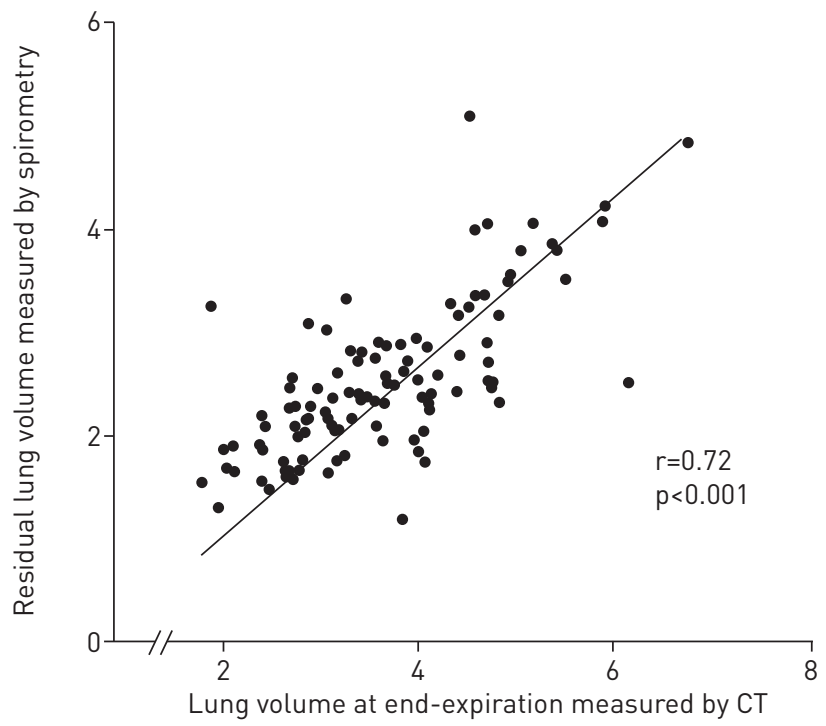

Comparison between COPD defined using fix ratio or $L L N$

Since seven of our COPD patients with FEV1/FVC $<0.7$ had normal LLN, we also calculated our data excluding these individuals. The differences between our study groups did not change in any aspects whether we used the fixed ratio or LLN for definition of COPD in our material.

\section{Discussion}

In the present study, we demonstrate that morphological changes in smokers' lungs can be detected visually on inspiratory and end-expiratory computed tomography scans. We describe two populations of smokers, with and without regional air trapping, for whom the latter group had a lower FEV1 and FEV1/ FVC, more emphysema, a lower BAL fluid recovery and a higher ratio of expiratory and inspiratory mean lung attenuation (E/I, "static air trapping”).

Due to limited resolution of computed tomography to depict small airways with diameter less than $2 \mathrm{~mm}$, some authors $[6,11,12]$ have evaluated air trapping and micronodules on computed tomography as signs of small airways disease. Previous studies based on visual assessments of air trapping on expiratory computed tomography have demonstrated a negative correlation between presence of regional air trapping and lung function in asthma $[11,12,26]$. Recent studies have implemented several quantitative methods for evaluation of air trapping $[10,17]$. We applied a frequently used method, the ratio between mean lung attenuation at expiration and inspiration $(\mathrm{E} / \mathrm{I})[10,15,17]$ combined with visual assessment of regional air trapping. Interestingly, our results show a reciprocal relation between regional air trapping and E/I, ("static air trapping") in smokers. Thus, the subgroup of smokers with regional air trapping had lower E/I, a higher FEV1 and FEV1/FVC, less emphysema and a higher BAL fluid recovery. The other smoking group, without regional air trapping, had higher E/I, resembling the COPD patients, with a reduction in FEV1/FVC and FEV1, lower BAL-fluid recovery.

The ratio between mean lung attenuation at expiration and inspiration (E/I), "static air trapping" is influenced by lung parenchymal destruction and airways obstruction; both jeopardising the subject's ability to expire properly. Smokers with a better preserved lung parenchyma should therefore be able to empty their lungs more properly; enhancing appearance of a typical patchy pattern of regional air trapping on end-expiratory computed tomography. Higher lung density on expiratory computed tomography, less emphysema, less airways obstruction and higher BAL fluid recovery in this group of smokers, despite of equal smoking burden, age and BMI, are findings indicating better preserved lung parenchyma [27]. They might respond to cigarette smoke in a different way, or they are in an earlier stage of disease development, i.e. predominantly small airways disease prior to parenchymal destruction [9]. Regional air trapping was present only in three COPD patients, which indicates a widespread presence of static air trapping due to emphysema and airways obstruction in these patients.

Regional air trapping was detected in $45 \%$ of the healthy never smokers which is in line with previous studies $[28,29]$. Air trapping in the healthy never smoking lungs might be a physiological phenomenon related to ventilation and perfusion and should be an area for future research. Urban air pollutions may also trigger subtle small airways inflammation, without obvious clinical significance. Nevertheless, regional 
air trapping in never smokers in the present study was an isolated occurrence without any correlation to any other measured variables.

Neutrophils and macrophages are the first line of recruited cells to the airways and are abundant in BAL fluid or surgical resected tissue obtained from smokers $[1,25,30]$. Due to the ability of neutrophils to produce elastase and other proteinases with tissue destructing potentials, neutrophils are considered as the major culprit cells causing emphysema $[1,31]$. However, some data suggest a preservation of alveolar walls in the presence of neutrophils rather than pure destruction [32]. Our results show a weak but statistically significant negative correlation between E/I and BAL-neutrophils which may indicate that smokers with regional air trapping have more neutrophils in their lungs and can, simultaneously, resist parenchymal destruction. Presence of a less aggressive neutrophil phenotype and better ability to tissue repair in this group may be a possible explanation. However, this association should be interpreted with caution since it is only seen in smokers with normal spirometry and relies on a few subjects with higher neutrophil concentration.

Remy-JARdin et al. [6] described micronodules in $27 \%$ and emphysema in $20 \%$ of smokers. These proportions are lower than in our study. Higher prevalence of micronodules and emphysema in the present study might be explained by a higher age and smoking burden. In a longitudinal follow up of the same smokers, the authors found that micronodules were replaced by emphysema [33]. These micronodules, likely representing inflammatory lesions, may thus be a precursor for discrete centrilobular emphysema. Interestingly, the number of subjects with micronodules was much lower in COPD than in smokers, suggesting that the micronodules may have been replaced by emphysema at this stage.

This study has some limitations. The inspiratory and expiratory computed tomography scans were not spirometry gated which may have influenced our measurements. However, we found a correlation of 0.72 between residual-volume measured with plethysmography and lung volumes measured with expiratory computed tomography which indicates an adequate level of expiration (figure 5). Furthermore, we did not use a scoring system for morphological changes that allows assessing degree and severity of air trapping and emphysema. However, we deliberately avoided a more complicated scoring system to reduce the impact of inter-observer variability and excluded trivial air trapping and emphysema.

Using quantitative and morphological computed tomography and BAL, we demonstrate that smokers with normal spirometry can be divided into two different groups in an early preclinical stage. Smokers with regional air trapping had predominantly neutrophilic inflammation and a higher FEV1 and FEV1/FVC with less emphysema and lower E/I. These smokers might be less susceptible to parenchymal destruction or investigated at an earlier stage of smoking-related lung injury. Our results highlight heterogeneity among smokers with normal lung function and may be of help for early detection of smoking related structural changes in the lungs.

\section{Acknowledgements}

The authors thank study participants and Helene Blomqvist, Gunnel de Forest, Margitha Dahl and Benita Engvall for excellent assistance (Dept of Medicine Solna, Karolinska Institutet and Karolinska University Hospital, Stockholm, Sweden).

\section{References}

1 Hogg JC, Chu F, Utokaparch S, et al. The nature of small-airway obstruction in chronic obstructive pulmonary disease. N Engl J Med 2004; 350: 2645-2653.

2 Berend N, Wright JL, Thurlbeck WM, et al. Small airways disease: reproducibility of measurements and correlation with lung function. Chest 1981; 79: 263-268.

3 Galban CJ, Han MK, Boes JL, et al. Computed tomography-based biomarker provides unique signature for diagnosis of COPD phenotypes and disease progression. Nat Med 2012; 18: 1711-1715.

4 Yanai M, Sekizawa K, Ohrui T, et al. Site of airway obstruction in pulmonary disease: direct measurement of intrabronchial pressure. J Appl Physiol 1992; 72: 1016-1023.

5 Hogg JC, Wright JL, Wiggs BR, et al. Lung structure and function in cigarette smokers. Thorax 1994; 49: 473-478.

6 Remy-Jardin M, Remy J, Boulenguez C, et al. Morphologic effects of cigarette smoking on airways and pulmonary parenchyma in healthy adult volunteers: CT evaluation and correlation with pulmonary function tests. Radiology 1993; 186: 107-115.

7 Pipavath SJ, Lynch DA, Cool C, et al. Radiologic and pathologic features of bronchiolitis. AJR Am J Roentgenol 2005; 185: 354-363.

8 Karimi R, Tornling G, Forsslund H, et al. Lung density on high resolution computer tomography (HRCT) reflects degree of inflammation in smokers. Respir Res 2014; 15: 23.

9 McDonough JE, Yuan R, Suzuki M, et al. Small-airway obstruction and emphysema in chronic obstructive pulmonary disease. N Engl J Med 2011; 365: 1567-1575.

10 Bommart S, Marin G, Bourdin A, et al. Relationship between CT air trapping criteria and lung function in small airway impairment quantification. BMC Pulm Med 2014; 14: 29.

11 Berger P, Laurent F, Begueret $\mathrm{H}$, et al. Structure and function of small airways in smokers: relationship between air trapping at CT and airway inflammation. Radiology 2003; 228: 85-94. 
12 Arakawa H, Webb WR. Air trapping on expiratory high-resolution CT scans in the absence of inspiratory scan abnormalities: correlation with pulmonary function tests and differential diagnosis. AJR Am J Roentgenol 1998; 170: 1349-1353.

13 Gevenois PA, de Maertelaer V, De Vuyst P, et al. Comparison of computed density and macroscopic morphometry in pulmonary emphysema. Am J Respir Crit Care Med 1995; 152: 653-657.

14 Chong D, Brown MS, Kim HJ, et al. Reproducibility of volume and densitometric measures of emphysema on repeat computed tomography with an interval of 1 week. Eur Radiol 2012; 22: 287-294.

15 Mets OM, de Jong PA, van Ginneken B, et al. CT air trapping is independently associated with lung function reduction over time. PLoS One 2013; 8: e61783.

16 O'Donnell RA, Peebles C, Ward JA, et al. Relationship between peripheral airway dysfunction, airway obstruction, and neutrophilic inflammation in COPD. Thorax 2004; 59: 837-842.

17 Hersh CP, Washko GR, Estepar RS, et al. Paired inspiratory-expiratory chest CT scans to assess for small airways disease in COPD. Respir Res 2013; 14: 42.

18 Coxson HO, Mayo J, Lam S, et al. New and current clinical imaging techniques to study chronic obstructive pulmonary disease. Am J Respir Crit Care Med 2009; 180: 588-597.

19 Ueda T, Niimi A, Matsumoto $\mathrm{H}$, et al. Role of small airways in asthma: investigation using high-resolution computed tomography. J Allergy Clin Immunol 2006; 118: 1019-1025.

20 Kohler M, Sandberg A, Kjellqvist S, et al. Gender differences in the bronchoalveolar lavage cell proteome of patients with chronic obstructive pulmonary disease. J Allergy Clin Immunol 2013; 131: 743-751.

21 Forsslund H, Mikko M, Karimi R, et al. Distribution of T cell subsets in bronchoalveolar lavage fluid of patients with mild to moderate COPD is dependent on current smoking status and not airway obstruction. Chest 2014; 145: 711-722.

22 Quanjer PH, Stanojevic S, Cole TJ, et al. Multi-ethnic reference values for spirometry for the 3-95-yr age range: the global lung function 2012 equations. Eur Respir J 2012; 40: 1324-1343.

23 Quanjer PH, Tammeling GJ, Cotes JE, et al. Lung volumes and forced ventilatory flows. Report Working Party Standardization of Lung Function Tests, European Community for Steel and Coal. Official Statement of the European Respiratory Society. Eur Respir J Suppl 1993; 16: 5-40.

24 Mets OM, Zanen P, Lammers JW, et al. Early identification of small airways disease on lung cancer screening CT: comparison of current air trapping measures. Lung 2012; 190: 629-633.

25 Karimi R, Tornling G, Grunewald J, et al. Cell recovery in bronchoalveolar lavage fluid in smokers is dependent on cumulative smoking history. PLoS One 2012; 7: e34232.

26 Verschakelen JA, Scheinbaum K, Bogaert J, et al. Expiratory CT in cigarette smokers: correlation between areas of decreased lung attenuation, pulmonary function tests and smoking history. Eur Radiol 1998; 8: 1391-1399.

27 Lofdahl JM, Cederlund K, Nathell L, et al. Bronchoalveolar lavage in COPD: fluid recovery correlates with the degree of emphysema. Eur Respir J 2005; 25: 275-281.

28 Webb WR, Stern EJ, Kanth N, et al. Dynamic pulmonary CT: findings in healthy adult men. Radiology 1993; 186: $117-124$

29 Tanaka N, Matsumoto T, Miura G, et al. Air trapping at CT: high prevalence in asymptomatic subjects with normal pulmonary function. Radiology 2003; 227: 776-785.

30 Bosken $\mathrm{CH}$, Hards J, Gatter K, et al. Characterization of the inflammatory reaction in the peripheral airways of cigarette smokers using immunocytochemistry. Am Rev Respir Dis 1992; 145: 911-917.

31 Ekberg-Jansson A, Andersson B, Bake B, et al. Neutrophil-associated activation markers in healthy smokers relates to a fall in DL(CO) and to emphysematous changes on high resolution CT. Respir Med 2001; 95: 363-373.

32 Finkelstein R, Fraser RS, Ghezzo H, et al. Alveolar inflammation and its relation to emphysema in smokers. Am J Respir Crit Care Med 1995; 152: 1666-1672.

33 Remy-Jardin M, Edme JL, Boulenguez C, et al. Longitudinal follow-up study of smoker's lung with thin-section CT in correlation with pulmonary function tests. Radiology 2002; 222: 261-270. 